\title{
CONSIDERATIONS ON THE EVOLUTIONARY THEORY
}

\author{
F.G. Brieger \\ August, 1983
}

It is not my intention to present a detailed history of the development of the theory of Evolution as generally formulated today, but only to present some steps which have had special importance in the past or which require now reconsideration. Some points of view accepted as essential in the past, owing to facts then considered evident, were not rarely included in the general theory though sometimes having resulted from bias, exagerating their importance while having actually become without much importance, thus representing a necessary ballast or dead weight.

Some mythological concepts about origin of life or more specifically of mankind, existed probably in many parts of the world and have been accepted by still rather primitive indigenous people. During the middle ages, ideas of Aristotelean philosophy or the biblical concepts were accepted as representing the "truth". In, the middle of the $18^{\text {th }}$ century modern or scientific reasoning started in the biological sciences, and one can, from then on, distinguish two phases of development of scientific study of evolution. In the first phase the questions of evolution as a process predominated, while after it's acceptance had spread more or less widely, the discussion passed to a second phase, the study of the evolutionary mechanism. Not infrequently both phases are called summarily "darwinism". Actually, 
Anais da E.S.A. "Luiz de Queiroz"

when DARWIN started his work and published the results, the hypothesis of evolution as a process had been accepted by many scientists, and DARWIN's contribution dealt mainly with the evolutionary mechanism, though greatlycontributing to strengthen support of the evolutionary hypothes is in general.

In preparing the present paper the question arose which bibliographical references should be given out of the vast number of publications existing today treating evolution and connected subjects. Such citations might help the reader, but would undoubtedly increase the size of this paper to such an extent that the treatment of some especially important points might become obscured. Thus oy only mentioning the names of the more important authors in the field, an experienced geneticist could easily find the necessary bibliographical reference, while for others the reading of specialized papers might be rather confusing.

The question of explaining the diversity of living organisms became important around the middle of the $18^{\text {th }}$ century, in view of the rather great number of taxa which had been accumulated in consequence of the expansion of world shipping and the exploration of colonial areas by the great European powers. Special methods of an archivistic nature were required to bring order into it and this was the great merit of LINNAEUS to have provided an orderly arrangement, establishing both firm concepts such as that of the species and basic nomenclatural rules for clear identification. Both, the species concept and the basic nomenclatural rules then established, are valid still today. LINNAEUS recognized that his system was an artificial one, but he soon came to the conclusion that there exists an underlying Natural System. The origin of the existing diversity did not represent any difficulty to him, since he accepted firmly the biblical interpretation and simply concluded: there are many species as had been determined by the Creator. The paleontologist CUVIER 
was especialiy interested in the observations that fossil species were always found only in a restricted series of geological strata and then substituted frequently by rather similar species. This extinction and the following new formation of species he also interpreted as acts of the Creator, but he added some new elements. He formulated the hypothesis that at different geological period the Creator became dissatisfied with his creations, provided a diluvium destroying all elements of flora and fauna, resuming then, the process of creation. Geologists did not find proof that such general catastrophic changes had ever occured. On the contrary, they found that some fossil taxa, specially species, disapeared, i.e., became extinct and then were substituted by new ones at some specific geological period, while in others such events had taken place at quite different geological moments. Furthermore, scientists may have come to reject CUVIER's hypothesis as fundamentally illogical and thus as inacceptable: it attributes to the Supreme Creator the human quality of using the simple method of "trial and error" repeatedly. In fact C.UVIER's hypothesis was not accepted as a valid explanation, not only because not having been proved, but by leading to too many contradictions. Thus an alternative explanation was required.

The general situation in the second half of the $18^{\text {th }}$ and the first of the $19^{\text {th }}$ centuries may easily have caused discussions of another nature. It was evident that the political system of the absolute monarchy had resulted in social misery and economic bankruptcy, requiring drastic changes. Some thought the only solution to consist in one, very drastic and revolutionary change, while others prefered a sequence of smaller changes without a general upheaval, i.e. the process of slow, but continuous evolution towards a preestablished end. The French followed the first mentioned way, that of the great French revolution, while in England and elsewhere the process of slow and gradual change was adopted. Similar thoughts may have 
played considerable role in scientific thinking. CUVIER's hypothes is of repeated diluvia correspondend rather to that of revolutionary change, while Auguste de St. Hilaire, LAMARCK and others accepted an evolutionary hypothesis. They postulated that taxa (species) were step by step transformed into new ones. No proof of this is available owing to the fact that such evolutionary transformations require evidently geological periods, much longer than life of one scientist or even that of several successive generations of scientists. But several series of fossils have been found in subsequent geological periods, showing stages in the development of some characters which can be interpreted as successive phases in an evolutionary chain, for instance the formation of the foot of horses, the trunk in elephants or others linking apes to Homo sapiens. In such series of fossils the species following each other are most probably not directly connected, but represent only steps in a general phylogenetic sequence. The species at one level need not to have been the actual ancestor of the fossil species of the following level. Studies of comparative morphology have also resulted in establishing what might be considered as successive steps in an evolutionary sequence even with regards to characters of higher taxa. The sequence from Pteridophytes over Pteridosperms and Gymnosperms to Angiosperms shows an almost continuous change. From the still autotrophic haploid prothallum of ferns it passes to the female gametophyte formed within the female tissue of the iiploid sporophyte. The male gametophyte may form in the Gingkoales a tube or haustorium producing still two mobile spermatocytes, until finally in the Gymnosperms such haustorium, now called pollen tube, transports the two male nuclei to complete fertilization of the oosphere. The decisive element allowing the evolutionary concept to be generally accepted consists in the complete absence of any evidence to the contrary. The evolutionary concept was consequently slowly accepted, mainly during the first half of last century and is still considered valid up today. 
As long as the biblical concept had been acceptable, there was no problem regarding the origin of life and the dogmatic statement: "omne vivum ex vivo" remained valid. Some concepts regarding the origin of living organisms had been formulated and formed part of aristotelean philosophy, regarded as fundamental since the Greek classics. During the last century, PASTEUR and others accumulated experimental evidence disproving the speculation that life could have originated by some combination of dead matter, thus at least apparently disproving the occurrence of "spontaneous generation". Such experiments, though without positive results, did not prove anything, since it was not shown that the experimental conditions had been the right ones. On the other hand, if the evolutionary hypothesis is a correct one, life must have started somehow, somewhere and at some time. Rather recently scientific results have indicated how and when life could have had its origin. Geological and petrographic evidence indicate that in the course of some 5 billion years the earth suffered important changes. In about the first half of this long period, free oxygen was either absent or extremely rare in the atmosphere which contained larger amounts of nitrogen, carbon dioxide and anmonia vapur, which form under some conditions, water soluble complex molecules dissolved in ocean waters or rivers. Such chemical combinations similar to proteins have in fact been produced experimentally. As soon however, as some photochemically active combinations similar or identical to chlorophyll were formed, the situation changed and free oxygen appeared in the athmosphere. This in turn would have caused the immediate destruction through oxidation of many or most "organic compounds" - so called by tradition, though not of organic origin unless they were already protected by special membranes. This might be considered as a possible origin of living organisms. Again it must be stated that there is no proof of this assumption, but it shows that such an origin might have taker place. 
So far I have tried to summarize why the continuous process of evolution has become generally acceptable. It was never proved, but there are no contradicting facts known and it is not only logical, but also in agreement with all facts known, in nature or from experiments.

Now I will pass to discuss the probable nature of the evolutionary mechanism which allows the permanent continuation of the evolutionary process. The concept of its nature which up to now widely was accepted, could be rightly called "darwinism" and shall be submitted to a critical analysis. However, before entering into this subject, LAMARCK's ideas about the role of the supposed inheritance of acquired characters must be mentioned rapidly, mainly for historicail reasons.

LAMARCK, a French biologist with a vast taxonomic knowledge believed that some characteristics in animals, including man, could not only be improved by intense training, but also become to be heritable in this improved form. However, even in human society there are probably more cases showing the contrary, i.e., of inefficient children of outstanding people not only in some special fields but also after special training. Experiments were carried out by several biologists, and they did not show any signs of an existence of heritability of acquired characters. Thus LAMARCK's hypothesis was rejected and finally abandoned.

DARWIN's way of thinking was probably predisposed by the general spirit in England at the time of the beginning of intense industrialization where both extreme competition between new industrialists and at the same time very strong exploitation of the labour forces were considered essential for progress.

Furthermore, he knew that in animal breeding, sudden heritable changes of characteristics did occur, at that time called "sports", now mutations. Finally, travelling as medical officer over the Atlantic Ocean 
and landing on the shore of Rio de Janeiro, he became aware of the richness of both flora and fauna of the tropics, so much contrasting with the comparably rather poor vegetation in England. Combining all these thoughts and observations, he concluded that also in nature there must exist an extreme struggle for survival and that any hereditary changes which increase the chances for survival would be selected rapidly through competition with their own predecessors. Thus the hypothesis of darwinistic "Natural Selection" was established. DARhIN delayed publication of his hypothesis until much later after having accumulated a great number of observations which he could interpret with his hypothesis. This was rather rapidly accepted by an increasing number of scientists and gained general acceptance as a satisfactory explanation as a mechanism of the evolutionary process, based exclusively on mechanical elements and free of any vitalistic or biblical concepts. Evidently, as was the case regarding the hypothes is of evolution as a process, no experimental proof could be furnished: evolutionary progress is so slow, requiring, geological time scales,but not some based on the duration of human life. With the intention, however, to provide "proof" for the darwinian hypothesis, his followers accumulated a very great volume of observations which were interpreted according to the requirements of the darwinian theory. In fact, the latter became a dogma: either observed structures or functions could be interpreted according to darwinism or they were simply disregarded. With this form of procedure a tremendous bias was introduced. Such bias has led to quite erroneous interpretations of characters to be of value for increasing survival capacity. An almost dogmatic belief in the ever presence of darwinian natural selection has caused even a teleological form of thinking. Every character of an organism has to serve somehow for one purpose specially: to improve survival capacity. Unfortunately this principle was not rarely used, interpreting only the functions of characters of living organism without proving that such an increase in survival capacity had been achieved. One of many 
examples only shall be mentioned in some detail. In the higher plants (Angiosperms) the function of the flora1 organs consists in allowing pollination. The structure of the floral organs has however also been used largely toestablish order in a "Natural System", i.e., one which corresponds to the changes which had occurred uuring evolution. Consequently, in applying the darwinian dogma, each special feature of floral organs must represent some improvement in the pollinating process and through it to an increase in survival capacity. Among the families of Angiosperms, the orchid family is often cited as showing the most astonishing "adaptations" between flower and pollinator, sometimes to a group of insects, sometimes to one specie or even to one sex of a specie only. The often complicated floral structures in orchids became a very special and often cited object of darwinistic interpretation. For instance, it was observed that they attracted by their scent, coloration and form the males of wild bees which, confusing the flower with a female of their species, tried to copulate with the flower and during these movements transferred the pollinia from the anther to the receptive stigma of the same flower, a procedure called "pseudocopula". The behaviour of the insect males was. clearly observed, but not that fruit setting followed always after pseudocopula. Other interpretations in flower ecology were rather anthropomorphic. For instance longitudinal dark stripes on floral leaf organs pointing somewhere into the flower were interpreted as serving as indicating direction as if the pollinator had learned some traffic signs, while ignoring transverse stripes indicating to human impediments of movement. It seems very doubtful that bumblebees will seltle one on top of the other, but a design on an Ophrys-flower, appearing to the human eye as an image of such an insect, has been interpreted as informing the insect that some special food or drink might be obtained, an impression analogous to that of a human person on the outside board of a bar or restaurant. However most important is the fact that generally no test is made to prove the efficiency of the 
adaptative relations between flower and pollinator. Having studied tropical orchids for several decades, I undertook numerous collecting trips in many parts of the American tropics. Since orchids are long lived perennials, they can be easily found and collected. Most, if not all, species of this family flower each at a specific time of the year. They were often found without flowers, but when cultivated under near natural conditions they showed that they produce flowers normally each year. Since the ovary is generally in a bud stage at flowering time, developing only further after a successfull pollination, reaching maturity after considerable time, i.e. after several weeks or mostly many months, one might thus expect that fruits in some stage of development should be found on the mother plant on its natural habitat at any time of the year and in abundance. On the other hand fruits were only very rarely found on the natural habitat. This then indicates very clearly that the adaptations between orchid flower and pollinator are very inefficient under natural conditions and thus do not in any way increase survival capacity. Only ocasionally a few species were found with all their flowers developed into fruits. A detailed study of such species under cultivation and constant observation showed that they had special, often minor structural changes responsible for automatic selfpollination besides all characters typical for each species or genus. Leaving aside the few exceptional cases of automatic selfing, the majority of orchids show the relative inefficiency of the adaptations between orchid flower and pollinator which rather represent a disadvantage and not an increase of survival capacity. Since however orchids have evolved and still exist,there must be some characters reestablishing efficient survival capacity and thus acting as what may be called a "compensating adaptation". Orchid fruits when mature can contain, varying from taxon to taxon, from several tens of thousands to some hundreds of thousand seeds. Since it would be sufficient for the preservation of a specie that one seed per parent plant would give a 
Anais da E.S.A. "Luiz de Queiroz"

mature specimen, the rate of survival under natural conditions must be an exceedingly low one and a large number of potential descendants are eliminated by some form of random elimination, independent from its individual survival capacity. This should not $h$. interpreted as indicating that there is no naitual selection of the darwinian sort, but that this is only part of the whole selective process and probably a rather minor part of it when compared with random selection. This conclusion will lead forcibly to reconsider many interpretations given to the evolution of characters which formerly have been considered as the result of darwinian selection. It will in no way affect the general acceptance of the evolutionary process as such, but only concepts regarding the evolutionary mechanism.

The science of genetics which has rapidly developed during this century, had furnished many contributions to the understanding of the evolutionary process and its mechanism, and thus, after changing the exclusive if not dogmatic basic concepts of darwinism it becomes necessary to enter into a discussion of pertinent cytogenetic questions.

When MENIEL discovered the rules of transmission and persistence of hereditary basic units, this was at first rejected, but CORRENS and others reestablished the mendelian rules. DE VRIES formulated his mutation hypothesis by erroneously interpreting results, but the existence of hereditary changes was later generally accepted. JOHANNSEN established aclear distinction between the carrier of the genetic information, carried out from mother to daughter cells and from generation to generation which he called the"gen" and on the other side the actual manifestation of this information the "phenotype". The fact that spermatocytes or pollen tubes transfer only and with regularity nuclei into the oocyte rarely also some small part of cytoplasm, made it evident that only nuclei are responsable for genetic transmission. Cytologists proved then that mainly the chromosomes of 
the various components of the nucleus were responsible for that transmission through the complex mechanical basis of mendelian segregations. Also the chromosomes have a complex structure. Sometimes living cells show that chromosomes contain a spirally enrolled thread or threads, the chromosomes, though it was long under discussion whether these remained intact during the resting stages of the nucleus. After treatment of living chromosomes (i.e. without fixation of any sort), these spirals could become visible without staining, but considering their thickness they leave a central space which must be filled by some material, the "matrix" forming the main body of each chromosome. In treating living cells during nuclear division by various chemicals (ammonia, nitrates of $\mathrm{Ca}$, Na, Li, etc) I observed (BRTEGER unpublished) that the chromosomes start to swell, chrononemas becoming visible within a colorless and uniform matrix which thus differs clearly from the surrounding granular cytoplasm. The swelling matrices touch and fuse finally while the chromonema unroll almost completely. Thus the final aspect differs only in so far from a nucleus in the resting stage that it has no outer menbraw nor a definite form. The functions of the matrix are unknown, but it certainly has no part in preserving a genetic information.

The chromonemas are rather long and thin threads, though of varying thickness nodules of varying sizes being connected by a thin thread. The pecularity of both mitosis and moiosis also indicate clearly that there exist some orderly sequence in the arrangement of gen loci, linked with each other fixed rates when located in the same chromonema. Attempts were made to determine the size of a gen locus. The total length of the chromonema can be measured with some accuracy. The total number of gen loci has been estimated for some species to be of the order of several thousands. 
A chromonema is composed of two chemically different parts: the DNA-chain, formed by longitudinal unions of only four kind of nucleotides and its protein cover. Since the DNA chain was discovered it was postulated that its parts should contain permanently the genetic messages in some coded form which then vill be activated and transmitted somehow and somewhere to be executed and transformed into phenotypic manifestations. It was assumed further that the four nitrogen basis in various numbers of all combinations and permutations would furnish the basis for the transmission of genetic informations of the numerous gen loci and the allels of a specie. However since the same nucleotides are present in all species, it seems to me very doubtful if they would be sufficient to build up so many different types of genetic messages. Before the discovery of the DNA-chain, it was accepted that proteins were the basis of what was first called a "mendelian factor" and later a gen. It would seem to me more logical to return to such an interpretation, considering that proteins would be a more adequate choice in view of their chemical complexity and diversity as the material basis of a gen locus and its various allels. The DNA-chain would then have the function to be the first step in a sequence transferring the information to other parts of the cell or other cells able to produce phenotypic manifestations. It really does not matter very much whether we accept the former assumption of the DNA-chain or that of its protein cooper as representing the conserver of the coded genetic information.

The conservation of the whole genotypic information in some parts of the chromonemas seems to be the last conclusion which can be applied in a general way to all organismswith normal mitosis and meiosis. Regarding if connection with the final phenotypic manifestations, there are some indications refering to special cases, but which represent and at present merely some form of pieces of a preliminary working hypothesis to be further tested. It must be remembered that the genotype as a 
whole represents a kind of master-plan which not only determines the different phases of ontogenetic development, but also the functioning in the adult. Though in some cases phenotypic manifestations seem to be controled by one or few gens only, in fact a number of modifier gens are also involved and in addition the manifestation depends on from influences from the outside. We do not yet know anything about when and how the genetic message is modified under the influence of external factors. It is equally unknown how certain parts of the genetic message are activated in some cells in time and space within an organism, though the whole message is present in chromosomes of all cells of that organism. Some authors have attributed activation to protein molecules of the DNA-cover, while others admit that hormones might be responsible. But what might activate these "activaters" at the right time and place only in some but not all cells? It is assumed that messenger-RNA, diffusing from the chromonema into the cell transports somehow the message to other parts of the cell such as the ribosome which then are able to decode the message and provides its phenotypic realization. But when no such special inclusions aro known to exist, what part does the decoding? How (ail an individual cell "know" what size, shape or function it has to assume? SPERMANN, HARRISON and their groups have established that a certain group of undifferentiated cells in embryos of Amphibians induce cells more on the outside to form normal eyes or legs anywhere in the embryo, in places to which they have been transplanted experimentally. How they activate in the induced cells the respective gen loci is completely unknown. In the process of regeneration of lost parts or the recovery of cuttings in plants, somehow the course of ontogenetic development or only parts of it are reinitiated in accordance to the genotypic master-plan. Again it is not known what determines which part is reinitiated. Evidently biologists have not yet discovered efficient methods of experimentation and observation to enable them to uncover the pathway or 
possibly the pathways linking the conserved genotypic master-plan with the phenotypic manifestations. It seems now indicated to review some other items which have been incorporated into the current evolutionary theory.

Up to the point of considering the chrus some as the carrier of genetic messages, science is still on a firm basis, but linking the genetic message to its phenotypic manifestation we have at present only incomplete and rather vague working hypothesis.

When T.H. MORGAN started his work in genetics he used as laboratory animal the fruit fly, previously found by CASTLE to be easily cultivated and studied in milk bottles. BRIDGES, then on $1 y$ a laboratory servant, though later a very distinguished geneticist, was told to collect fruit flies in orchards, fruit shops and elsewhere, to cultivate them and see what variations he could find differing from the common or "wild type". He carried this work very efficiently out, isolating individuals with aberrant characters, tested them in crosses and kept only those which gave clear mendelian or near-mendelian segregations in such test crosses. All other aberrant forms were simply discarded. This method seemed to allow to reach general conclusions regarding the nature of heredity, but involved a very strong bias regarding the general nature of mutations: namely that they were generally recessive and reduced survival capacity. Genetic research with mice, rats, rabbits, poultry or with maize, peas, sweet peas, snap-dragon, etc., confirmed that initially mutant genes were generally recessive, but not always subviable.

NILSON-EHLE and CAST, studying the heredity of quantitative differences, concluded on the other hand that such differences were controlled by rather numerous gen loci the allels of each locus when heterozygous being intermediate between the homozygotes in their phenotypic manifestation and had no effect on survival quality in any combination. The analysis of the mendelian 
proportions of monofactorial segregations of this nature is mostly difficult since the distinction of the three monofactorial genotypes are not very sharp owing to graduations of their phenotypic expression.

\section{other cases must be considered where the} phenotypic manifestation of the heterozygotes go beyond the range of variation of either homozygote, the original one and the mutated one. A heterotic monofactorial effect has been studied in two cases, the colour of the feathers in poultry and the flower colour in some cultivated form of Silent armeria. These cases have been interpreted attributing the phenotypic effect to two extremely closely linked gens in the mutant, one a recessive inhibitor and the other a dominant intensifier which however is hypostatic with regards to the inhibitor when homozygous. The recessive inhibitor being inactive in heterozygotes, the dominant intensifier manifests its phenotypic effect plainly. In the case of sithm which I used during many years in classwork for students, many thousands of $F 2$ plants were analyzed without any case of a linkage break which might have proven the basic hypothesis. In maize or man as in several other species heterosis is caused through heterotic interaction of many gen loci. The use of hybrids with a maximum of strongly heterotic pairs of allels, resulted with inbreeding through autogany or brother-sister matings in a desastrous loss of vigour caused by the appearance of homozygosity even only in some gen loci.

As last case the possibility shall be mentionned of a mutant having a dominant effect. It seems that in such cases the mutant reduces survival capacity considerably. Such cases have been rarely observed and studied, for instance by R.A. FISHER in poultry. Apparently the extremely strong phenotypical effect of a dominant mutant causes a generalized unbalance in the genotype. 
From the foregoing considerations one must conclude that there exists no general rule for mutant allels to be always for even mostly recessive and to reduce generally survival capacity. It should also be remembered that no gen locus will cause specific phenotypic manifestations which are not affected by the presence of allels of other gen loci. These may act simply as modifier only, i.e. their phenotypic manifestation consists exclusively in modifying the effects of allels of other gen loci and which can easily reestablish lost survival capacity. Geneticists and breeders have used these possibilities by changing the modifier effects by carefully planning artificial selection.

The term "wild type allel" was used to identify allels present with very high frequency in natural population or sometimes in old and well established races of cultivated plants. It must however be remembered that the "wild type allels" must in turn have appeared at some time during evolution or breeding and represented once mutations. Thus the question arises how a mutation with its initial extremely low frequency might become as frequent as present day "wild type gens".

In the preceding paragraphs criticisms are brought forward again a certain monopoly attributed to the purely darwinian concept as explaining the evolutionary process. Thus it becomes necessary to reconsider in some detail the factors which are involved during this process. Its basis consists evidently in the substitution of the allels present at a given gen locus by a new mutant allel which causes a new way of phenotypic manifestation. This manifestation is always quite unpredictable and one can expect that initially it causes in the genotype as a whole a certain unbalance. Its frequency is necessarely at first extreme1y low, but then increases successively during many generations, substituting the original allel and possibly eliminating it completely. When analysing this change in organisms living today, it has become the custom to designate the allels present as "wild type 
allels" in contrast to the infrequent new mutant allels. The present "wild type allel" on its part must have originated at some previous time as a mutant and has substituted the preceding original allel. This part of the evolutionary process, the substitution of preceding allels is however so slow that it cannot be observed by direct observation. But such changes have been caused through artificial selection carried out by geneticists and breeders. An analogous result was evidently reached by natural selection which consisted both in random and in darwinian selection as explained already above, the first a consequence of the highly excessive offspring of each plant, well beyond the minimum necessary to substitute its progenitors and the second a result of differences in survival capacity. In order to visualize how the evolutionary process might have progressed, it became necessary to work out mathematical models of population genetics, establishing certain indispensable premisses which shall now briefly be discussed.

Mutation Pressure

The mutation rate is generally very low and if we accept a value of $u=0,001$ as an upper maximum this would evidently represent an unusually high one. It would appear at first in the heterozygous state and the formation of a homozygote might depend on the occurence of a second mutant allel for which the expected frequency would be still smaller, being equal to $u^{2}=1^{-6}$ within the same progeny and without intercrossing. If we assume however that such a mutation would occur in $\mathbf{n}$ individuals with homozygotes being formed through intercrossing, the total frequency of the mutant might rise appreciably though hardly reaching a value of more than $1 \%$. Even this very slow increase might be considered sufficient since we discuss events in geological time which are generally counted in millions of years. 
Number and Interaction of Allels Per Gen Locus

Next we must consider the number of allels per gen locus. Experimental evidence indicates that most frequently there are only two forms of phenoty ic manifestations per gen locus. This may either mean that only two types of mutations were possible, each determining one of two phenotypes, or that there might exist any number of mutant allels but only two forms of manifestations.

In accordance with the nature of interallel interaction there may exist three genotypes with the mendelian proportions of $(1: 2: 1)$ or two with the proportion $(3: 1)$. This means that the number of phenotypes would be reduced to two, one of which with three times the frequency of the other.

Non-allelic Interactions

If the allels at each gen locus should not interact with each other, the maximum of phenotypic types with $\mathbf{n}$ gen loci would be either $3^{r}$ or $2^{r}$. This number would be further reduced in those cases where allels of different loci should interact in such a way that various genotypes would manifest identical phenotypes. As an example cases of such interactions will be mentioned starting from a bifactorial proportion of $(9+3+3+1)$. In case of a cumulative effect of the number of dominant allels, the proportion might be changed to $(9+6+1)$. In case of complementary interaction requiring at least one dominant allel in each of the two loci, the result would be a segregation of $(9+7)$ or when both loci have to be homozygous recessive $(15+1)$. The 9 possible genotypes of a bifactorial segregation with dominant allels in both loci would be reduced to 4 phenotypes and then further to a minimum of 2 phenotypes by interallel 
interactions. Considering the generally very high total number of gen locci, such reductions would still preserve a great deal of diversity in populations of a specie, but phenotypes based on several genotypes would have a frequency equal to the sum total of the individual genotypes including heterozygotes and thus be considerably larger than that of any genotype individually.

Random Selection

Random selection is an automatic consequence (a) of the fact that on the average each individual of a population produces more offspring than would be necessary to maintain population size; (b) of the existance within a population of numerous phenotypes with different frequencies and (c) in consequence of differences in survival capacity. In the latter case the following should be explained: if a mutant allel causes a rather weak manifestation and this only when homozygous then it may cause a certain unbalance of the genotype resulting in lower survival capacity. If the penetrance should be considerable, the mutant allel may manifest its phenotypic effect already in the heterozygote and thus act as a dominant allel. In this case the unbalance of the genotype will also be much stronger. In fact rather few such dominant mutants have been observed in experimental work. Finally if a mutant has very little penetrance, it can be considered as a neutral allel and will hardly affect survival capacity. Generally the action of individual mutants of this type cannot be analyzed individually and they are thus mainly considered as modifiers of the action of other mutants with a stronger penetrance. In fact selection of such modifiers may affect and reforce the penetrance of other mutant allels, and remove subviability. This last step will however not be anymore the result of random selection which, by definition, is without direction, depending exclusively on the frequencies of phenotypic 
manifestations of mutants. It may result in the preservation of some of them within the population and the accidental elimination of others in the next following generation. Diversity in the population will be reduced and homogeneity accordingly increased.

Ecoselection is a term which I propose exclusively to indicate selection caused by interaction between phenotypes, resp. their genotypes present in a population, and the ecological conditions under which the population lives. One can distinguish three different situations:

a) Some phenotypes may neither be advantageous nor disvantageous under the existing conditions and thus may be considered "neutral", not suffering darwinian selection, except at most in the form of a modifier shift, stabilizing the phenotypes and compensating any eventually existing subviability. Since such manifestations must have resulted under random selection reference to them was already made in the preceding paragraph. Thus they do not form any type of adaptation.

b) Other phenotypes may be advantageous under existing ecological conditions and their frequencies might be increased under darwinian selection. Consequently they might be considered as adaptations.

c) Finally some phenotypic manifestations might be unfavorable under the existing conditions of the environment and consequently will suffer under darwinian selection and may eventually eliminate from the population.

The phenotypic manifestations and their genotypical basis have been established under random selection and are completely independent from each other. Ecoselection may affect them individually. The term "adaptation" does not refer to some situation resulting from selection, but something which existed incidentally after random selection. This situation has been called by some 
authors "preadaptation".

DARWIN established the principle of the "survival of the fittest", though originally he used the terms "of the strongest". His followers tried consequently to interpret as many characters as possible attributing to them special adaptative value in increasing favorably their frequencies. This has led many to use even a certain form of teleological thinking, presenting their interpretation of facts, but not proving that all characteristics thus interpreted in fact improved survival capacity. In an earlier part of this paper I tried to show that a proof of any darwinian interpretation is absolutely necessary and that for instance the adaptations of orchid flowers to specific pollinators are generally inefficient. Survival capacity is only restored through another, but completely independent adaptation guarantying an excessive number of seeds in each of the few fruits.

We can now draw the following conclusions:

I. All the items so far mentioned change somehow the frequencies of genotypes, respectively of the phenotypes they cause, within a natural population in favor of the more frequent ones.

1I. The different factors enter sucessively into action in the following order: pressure of recurrent mutation rate, interaction between allels at the same or at different gen loci, random selection and finally ecoselection, but they remaining then in action continuously.

III. As a result the population will have characteristics which are either neutral, i.e. are not-adaptive with regard to the ecological conditions present, or can be considered as adaptations to these conditions, besides an evolutionary potential gen reserve, mainly formed by modifiers with rather weak phenotypic effects. 
IV. The term ecoselection is used in a different way from the originally, proposed by DARWIN. Adaptations are not produced under the principle of a "selection of the fittest" but are present as a result of random selection.

V. Ecoselection does not alter the frequencies of neutral characteristics present, it may increase the frequency and expression of adaptive characters, but it will reduce the frequencies of unfavorable characters or even eliminate them, unless they have been compensated by other and independent characteristics.

The items which now will be discussed hardly affect the frequencies of the different allels in the genotype but can influence strongly their homozygosity and heterozygosity.

The Mode of Sexual Reproduction

The mode of sexual reproduction is another important element in establishing models of population genetics. It has been also an object of special interest to taxonomist who used the characters of reproductor largely in distinguishing taxa at different levels. Also DARWIN and his followers saw their importance and studied the modalities of sexual reproduction specially from their point of view. Somehow the opinion was accepted that there are two alternative forms of sexual reproduction: allogamy and autogamy. On the other side I came to the conclusion that they are not mutually exclusive alternatives, but that there are quite frequently mixed forms of reproduction in various degrees of mixture between two alternative extremes. The question can be easily solved by a simple experimental arrangement, using plant species with hermaphroditic flowers, the male and female organs maturing at the same time. 
One plant of a specie carrying a genetic marker is to be planted in the middle of a number of other plants of the same species but carrying another marker. The F1 sometimes the F2 from seeds of the central. plant 'will then show whether it was self-pollinated or outcrossed and the percentages of both can be determined.

There are also other indication of the occurrence of mixed pollinations. Nicotina tabaccum has the typical flower of a species adapted to insect pollinization: the flowers are attractive bright pink and produce nectar abundantly. Nevertheless even an isolated plant, well protected from pollinators, will produce well filled capsules from all flowers. Though it has the "adaptations" of an insect pollinated species, its anthers are arranged tightly around the stigma and shed their pollen abundantly at the time when the stigma is receptive.

Another interesting case was observed at the John Innes Institution when I started work there. The flowers of the sweet pea are not automatically selfpollinated. The anthers form a close cycle with the pollen sacs slightly above the stigma in the middle. The corresponding petal is doubled up along its midnerve, with its sides tightly pressed together and only a small opening at the tip. Rubbing this petal between ones fingers, the flowers were selfed. If on the other hand, a common bee settles on the petal its weight forces the petal to move somewhat downwards and this movement causes pollen grains out of the pollen sacs and on to the stigma, also causing autogamy. However if a representative of a larger and heavier species visits a flower, its heavier weight forces the petal much further down exposing the stigma. If the large insect has visited other flowers previously and collected pollen placing it in a kind of basket on his legs, then it may happen that some foreign pollen grains fall out of the basket on to the stigma, causing a certain degree of allogamy, in the present case considered as contamination. Thus the species seems to 
be adapted to one pollinator species promoting autogamy, but when visited by another pollinator produces mixed seeds.

Next maize shall be mentioned, a wind pollinated species with separate male and female flowers o.t the same individual. Maize is also called as being "panmitic" since here the pollen is not carried by a pollinator from plant to plant, but first the pollen of many plants is mixed in the air by wind and lighter air currents, before it slowly falls downwards owing to its weight. The male flowers are formed at the top of the plant in the tassel, while the female flowers of the ear stand about in the middle of the plant in the axil of a leaf sheath, protected by a number of additional sheaths and only the stigmas, the silk, hanging out of this cover. Even if pollen grains could fall vertically down, they will generally not reach the silks which are protected by the leaf blade above, leaves being arranged in two rows, to the right or left of the main stalk, on either side each leaf above the next one lower down. Thus it seems that the whole arrangement is an adaptation to allogamy. However if one observes plants growing in an isolated place, far from other maize plants one will find that their ears contain generally some kernels which thus must have come from autogamy. Thus in panmitic maize, allogamy present dominates but does not exclude occasional autogamy.

Though possibly or rather probably some mixture between allogamy and autogamy are common in hermaphroditic species, in bisexual ones of animals or plants crossing between closely related specimens such as brother-sister-matings may take effectively the place of autogamy as a rather close approach. From a general point of view we can consider exclusive allogamy or exclusive autogamy as extremes of a wide range of mixed sexual reproduction. This is important since these two extremes will necessarily cause quite different results. Under allogamy heterozygotes are present as the result of combination 
at the beginning of every new generation, while under autogamy the number of heterozygotes will be automatically halfed in each generation so that ultimately no heterozygotes will be present, except some resulting from new mutations.

This will however affect the general situation considerably, in view of the fact mentioned above that many phenotypical manifestations are of a polymeric nature and thus require the combination of several mutated gens in different chromosomes. In exclusively autogamous species intercrossing between individuals not taking place, the population will consist of several homozygous genotypes and many combinations of mutants at different gen loci will not be present. This would certainly restrict the possibilities of evolutionary changes and might justify the opinion of many authors that autogamy is a "blind evolutionary alley". But even if autogamy should be in some species absolute rare intercrossing may still act as a compensating factor. In fact, there is no question that strictly autogamous species have evolved and exist. This is another indication that absolute autogamy can only exist when at least ocasionally substituted by some intercrossing within a population.

Hÿbrid Vigor

It was just discussed that some degree of autogamy will reduce the frequency of heterozygotes in a population, thus it will be indicated to discuss special situation in which the genetic basis causes an increase of vigor and thus affects favorably the frequency of heterozygotes. The necessary genetic basis may result from two special situations: cumulative interaction of allels at different gen loci or a heterotic interaction of two allels at the same gen locus. The first may be responsible when hybrid vigor becames manifest in crosses between unrelated 
populations within a specie or in interspecific hybrids, as a result of the accumulation of allels which when incidentally combined had a cumulative effect. In the second case however one has to assume that within one population mutant allels with a heterotic interaction had become accumulated. Some authors discussed $i{ }^{2}$ te past whether the last mentioned genetic formula coild be accepted and they postulated instead that the two allels in question should be considered as belonging to two different gen loci which however were absolutely linked. Even in the very few cases where a heterotic effect was caused by one pair of allels at one gen locus, no support for this assumption came forward. In one of such cases that of Silene armeria where the monofactorial heterozygote has deeper coloured flower than either parent line. I used heterozygotes as material for student class work during many years. F2 segregations were always in agreement with the ratio $1: 2: 1$ and no break of the supposed linkage was ever observed in several hundreds of F2 generations.

By definition homozygotes will have mostly less vigor than heterozygotes both in the case of cumulative effects of allels in different or heterotic effects of allels at one locus. In the case of cumulative interaction, the situation might be explained using a bifactorial mode1: AAbb $x$ AaBb. Such a heterozygous hybrid would in fact give homozygotes of the formula $A A B B$, consequently as vigorous as the double heterozygote $A a B b$, but with a frequency of only $1 / 16$. Since there is no reason to admit that only two loci might be participating in causing hybrid vigor, the frequency of such multiple homozygotes will be much smaller and might become practically insignificant. In the case of heterotic allels reduction of vigor caused by homozygosis will be unavoidable by definition.

Next question shall be briefly discussed how gene causing hybrid vigor might become established during the evolutionary process. Since the formation of mutants 
with various phenotypic effects is a matter of chance, there is no reason to reject the possibility of two mutations occurring independently, which however when incidentally combined might result in a cumulative effect such as hybrid vigor. The situation is on the other hand different regarding heterotic pairs of allels. These might easily be preserved in predominatly or exclusively allogamic populations where heterozygotes are formed automatically in each generation. In predominantly or exclusively autogamous population the situation will be quite different. In these the number of heterozygotes is automatically halfed in each generation through autogamy and thus heterotic pairs of allels could only be preserved when they cause such an extremely strong vigor able to compensate the loss through segregation. This then would explain why heterosis is absent whenever allogamy predominates. Hybrid vigor is not to be considered as an "adaptation promoting allogamy", but as its consequence.

Self- and Cross-Incompatilility

Self- and cross-incompatibility are both interpreted as possible adaptations promoting somehow either allo or autogamy. In fact however it seems to me rather more correct to consider them not as causes favoring one or other form of sexual reproduction but as effects of the latter.

Gens for self-incompatibility are not too rare in species of hermaphoditic plants. If they should arise by mutation in a predominantly autogamous species they will have little chance of being preserved under such conditions while nothing will hinder their presence in predominantly allogamous populations. Such a selfincompatibility might either be caused sporophytically or by an interaction between the female organs of the mostly diploid sporophyte and the generally haploid 
male gametophyte. The latter is the cause of the S-allels analyzed in details by EAST and his coworkers A. MANGELSDORF and BRIEGER. These $S$ factors represent a mixture of both self- and cross-incompatible genetic elements. In a cross between two plants carrying one identical allel for instance $S_{1} S_{2} \times S_{1} S_{3}$ the $S_{1}$ pollen tubes do not function normally but have their growth stopped in the style. If both allels should be different $\mathrm{S}_{1} \mathrm{~S}_{2} \times \mathrm{S}_{3} \mathrm{~S}_{4}$ all pollen tube grow normally and in crosses $S_{1} S_{2} \times S_{1} S_{2}$ or after selfing all tubes stop and no seeds are formed at all. Since the s-allels form a rather long series or multiple allels in natural populations, the eventual cross-sterility of some combinations do not affect seriously the frequency of individual allels in predominantly allogamous populations, though in autogamous populations such allels arising by mutation hardly will have a chance of preservation. It must also not rare in species of hermaphroditic plants, but their action is frequently quite incomplete. Thus in Nicotiana sanderae, the $\mathrm{S}-\mathrm{alle}$ ls are quite inactive in buds and thus in flowers when just opening as also in the last flowers formed at the end of the flowering period of a panicle.

The three factors just discussed, mode of sexual reproduction, hybrid vigor and self- or crossincompatibility hardly affect individual gen frequencies, but will alter the frequencies of phenotypic character manifestations and thus indirectly that of their genotypes whether heterozigous or homozygous.

Evolution and the Distinction of Taxa

Evolution and distinction of taxa represents the last subject to be discussed. Taxonomy or Systematics whatever designation one may prefer, is the oldest branch of biological sciences which was started in the middle of the 18 th century, well before any evolutionary 
idea had been expressed. Linnaeus's first task consisted to bring order into the vast material to be studied. He noticed very clearly that the enormous diversity of organisms is not a continuous one. This led him to distinguish very sharply two categories which he designated as those of the genus and of the species giving already more weight to the latter.. The origin of such categories did not represent any problem to him since he was firmly convinced that the biblical thesis was correct and that there existed only those species as created by the supreme intelligence. With the acceptance of the evolutionary concept new problems arose which are partly not yet very clearly resolved. Some authors up to recent times try still to consider the species as a real and quite distinct entity. Thus DOBSZHANSKY and others postulated that species must always be separated by "barriers of reprodutive isolation" and tried to explain their origin in the following way: a specie, though initially occupying one continuous area, came somehow to be distributed over two quite different and separate areas. When this possibility had to be rejected, it was assumed that it became adapted to two different ecological niches within one geographical area. In both cases it was then assumed that intercrossing between such separated populations became impossible and that in consequence each accumulated different mutants which when later crossed caused mutual incompatibility between components of the two separated populations. Thus a barrier was established and the two populations could now be considered as two separate-species. This speculative construction can be discarded, since the basic assumption of the obligatory barrier between species cannot be accepted. In many groups of animals there exists in fact a complete incompatibility between species as usually classified, while in few other groups of animals and in most higher plants not only interspecific but also intergeneric hybrids occur in nature and also result from experimental hybridizations. The result of such crosses is to some extent unpredictabde and may vary from complete failure to obtain even hybrid embryos 
Anais da E.S.A. "Luiz de Queiroz"

to the other extreme of obtaining Fl-plants with full fertility. J.CLAUSEN and others have attempted to use such degrees as a measure of taxonomic affinity between species or genera procedure which dependes again essentially on purely subjective reasoning. Simlar gradual differences exist between normally de ioping mutants over subviable ones to lethals.

The conclusion that reproductive barriers are not obligatory for separating species but they do not exist rarely. This forces one to ask inversity: how can such barriers be formed during evolution? The s-allels already mentioned above cause both self and crossincompatibility within populations. Heterostyly in some plants such as primula also causes a certain form of cross-sterility. There are two groups of plants present, some with a long style and thus with the stigma in a high position while the anthers are inserted below in the corolla tube, and others with a short style and thus the stigmas in a low position with anthers inserted higher up in the floral tube. There is a strong degree of sterility between plants within each group and full fetility in crosses between plants of different groups. In analogy one might postulate that mutants might occur in a heterozygote state $\mathrm{Cc}$ without having any effect in an adult organism, but mutually unable to form zygotes when combined in reproduction. Thus all individuals of the constitution $\mathrm{C}-$ as those of constitution $\mathrm{c}-$ are interfertile while no zygotes result from combinations of $\mathrm{c}-\mathrm{x} \mathbf{c -}$. The population will then be split into two intersterile parts and each part might accumulate independently different mutations.

In view of the absence of any objective criterium to delimitate the range of variation both within and between species or genera, the taxonomist has to evaluate subjectively the degrees of similarity or disimilarity between taxa. This method depends very much upon personal bias and consequently opinions of different authors not rarely disagree with each other though it is 
also understandable that different authors decided to use different characters in evaluation diversity. At first the differences in external and internal structural elements and their functioning were chosen to estimate degrees of differences and this method still represents the basis of taxonomy. Later on chemical differences were included. With the growing amount of genetical knowledge, the possibility offered itself to form opinions over the nature of the genetic basis of characters and judge thus at least apparently the progressive increaser of differences in genetical constitution. Differences in the pairing of chromosomes during meiosis were accepted as a criterium though this required the possibility to obtain at least interspecific hybrids. Rather recently it became possible to go even further isolating individual chromosome combining some obtained from different species and then verifying how intense this pairing was by determining at what temperatures $50 \%$ of the chromosomes unpaired. SIBLEY and AHLQUIST used this method in a study of a very large number of Australian birds. In part they confirmed the decisions of other taxonomists, while in other cases they suggested new forms of classification. The authors assumed arbitrarily that a lowering of temperature determining losses pairing of chromosomes would indicate degrees of lower evolutionary affinity. However it is not known that other factors but evolutionary affinity do in fact control of the pairing intensity. It should be remembered that a mutant allel has been found in maize which inhibits pairing during meiosis even in the presence of ten pairs of homologous chromosomes.

One can only conclude that there does not exist an absolutely sure method to judge or measure evolutionary offinity and that it depends on personal subjective judgement to which method each biologist might be inclined to give more weight in case of contradictory results.

On the other side it is quite evident that variation of characters of any sort is not continuous 
one, but that there exist differences of various dimensions between the so-called taxa. The decision made by LINNAEUS more than 200 years ago was a correct one from a practical point of view. His decision to establish the ranks of species and genus was fully justified and remains valid with the inderstanding that these ranks are conventional and thus arbitrary units. Nevertheless some informations regarding evolution can be obtained from taxonomic data. I have studied during several decads the taxonomy of orchids which represent the family of higher plants with the maximum degree of diversification as measured on the basis of the number of species and genera described. The great majority of the 20 to 25.000 species is composed of clearly defined units with a minimum os internal variability while a comparatively small number is so polymorphous that they had to be divided into subspecies or even lower ranks. An identical situation exists regarding about a dozen genera of a total of more than 800 . They contain more than 100 to over 500 species and can clearly be divided into a number of subgenera though some of them can very well be considered as separate genera. It cannot be decided whether such polymorphous groups are for some unknown reasons subject to a greater tendency for diversification or species, resp. genera still in active evolution while others have passed such a phase, unable to furtherdiversify and determined to eventual extinction.

The impossibility to define in an objective way what should be considered as "a specie" leaves only the conclusion that "the species" is not a fixed unit of any sort. It is in fact a stage within the evolutionary process which progresses slowly and might appear static for a limited period of geological time. What taxonomists call "a specie" corresponds to a segment of geological times corresponding in a way to an isolated snapshot taken out of a lost, but originally long cinematographic film. Speciation requires first the accumulation of a large number of mutants and this will be rather slow 
both under recurrent mutation rate and random selection. Considering that geological times are measured in millions of years, one may estimate that in one million years there may exist the following numbers of generations: about 35 million generation in the fruit fly one million in annual plants, some fifty thousands in longer living organisms like man or many trees which may start reproduction after some 20 years. Such high numbers of generations should be sufficient for even slow evolutionary progress. SIMPSON has given more detailed informations about this matter, specially accentuating that evolutionary progress has taken place at quite different rates in different taxonomic groups.

In spite of the impossibility to define the species concept-like those of a genus or still higher taxonomic levels-in a definite and objective form, it still represents a usefull and indispensable tool in all branches of biological science, necessary the material with one works.

\section{CONCLUDING REMARKS AND ABSTRACT}

Initially a clear distinction is made between the evolutionary process and its mechanisms.

It is perhaps not surprising that from time to time renewed attention is being given to what CUVIER more than two hundred years ago considered as evolutionary revolutions and which is now revised in the modern form of "punctualism". The gaps in fossil records and between the highest ranks of living animals are certainly impressive and still are unexplained. Consequently they remain open for more or less speculative interpretations. The main trouble consists in the lack of secure data from past geological periods regarding "missing links" 
which might or might not have existed. Thus only subjective interpretations are possible to explain those gaps. In plants such problems seem not to exist and the evolutionary sequence if not from Algae, at least from Pteriodophytes to the highest Angiosperms seems io be reasonably continuous, partly through fossils, partly based on studies of comparative morphology of still living intermediaries. Regarding speciation or what R. GOLDSCHMID called "microevolution", a hypothesis with respect to its evolutionary mechamism has been exposed in the present paper. The time rate of evolution has been studied by SIMPSON in detail and found extremely variable in terms of geological times, and the rate of evolutionary radiation is equally different in different groups, both including gaps or missing links. Such gaps may be considered larger in animal taxonomy. Without evidence regarding the existance or non-existance of such larger missing links, there seems to me no reason to assume some special evolutionary mechanisms for higher ranks or for what GOLDSCHMID has called "macro-" and "mega-evolution".

There are no doubts regarding the evolutionary process as such. It started some 2.5 billion years ago when under special external conditions complex water soluble chemical compounds, similar to protein and other "organic" substances were formed. The process then continued permanently forming first simple one-celled organisms up to that of complex multicellular ones.

The evolutionary mechanism requires still a careful and critical analysis. Two main theories have been proposed. LAMARCK postulated that there sould exist and inheritance of acquired characters but this was rejected owing to proof to the contrary. DARWIN'S started from the observation that orgamisms suffer ocasionally, though rarely heritable changes, at his time called sports, now mutations, the nature of their phenotypic manifestations being unpredictable and in no relation to any directed progress nor automatically 
advantageous under ecological conditions. Furthermore DARHIN postulated that these mutants became subjected to natural selection. Which led to competition between them resulting in the "survival of the fittest". Among the great number of such darwinian interpretations, the relation between orchid flowers and their pollinacors are cited. The rarity of fruit formation in the majority of tropical orchids show clearly that these adaptations are highly inefficient and instead of increasing survival capacity reduce it to such an extent that only another and unrelated character, the extremely high number of seeds per capsule reestablishes survival capacity. Thus the essence of the darwinian concept nust be rejected. Actually this has already been the case though in an indirect form. It became evident that adaptations in general require the accumulation of numcrous mutant gens before they really are of value increasing survival capacity. Their accumulation must thus precede any increase at survival capacity it is a consequence, i.e. their must have been an accumulation preceeding phase of "preadaptation".

The increase of mutant allels is first caused by recurrent mutation rate. Interactions between allels both at the same and at different gen locus reduces the number of different phenotypic manifestations, increasing thus the frequencies of those manifestation which are based jointly on different genotypes. The differences in frequency on the one hand on the other hand the excessively large number of descendants of organisms in general far in excess over the necessary for preservation, will cause a strong elimination or random selection.

The more frequent phenotypes have a better chance for being maintainer than the less frequent one. Some of them cannot be interpreted as "adaptations" but are rather of a neutral character. Others might be considered as adaptations favorable under existing ecological conditions; finally some might be in disaccord with the existing conditions and thus will have their frequency 
Anais da E.S.A. "Luiz de Queiroz"

further reduced up to complete elimination by what might be called "ecoselection", which I also have called in the Lext "darwinian selection". These different factors will enter into play in the order they have been mentioned, but will continue to act jointly, during a considerable time. The evolutionary process might come to in end when all probable mutations have been incorporated or eliminated from a population. However there remains always the possibility of a mutation occuring with an entirely new phenotypic effect and which thus may start a new evolutionary cycle.

Besides the factors already mentioned some others may affect mainly the relative frequencies of homo- and heterozygotes. The mode of sexual reproduction is probably the most important and probably the most frequently discussed one. It is pointed out that autogamy favoring homozygotes and allogamy favoring heterozygotes represent only the extremes of a wide range of combinations of mixed reproduction. Selfincompatibility and heterotic gen interactions also favor heterozygosity.

As a conclusion of the paper the delimination of taxa, specially of species is discussed. It is shown that there is no method yet available to define the species concept objectively. It remains however evident that variation is discontinuous and that such discontinuities can be used to distinguish taxa at different and subsequent levels. In general the lowest level receives the classification of "species" which thus represents a conventional and practicaly indispensable unit. The great majority of these species is well defined by their relative uniformity within and the descontinuity between these units. Only a small percentage offers difficulties owing to polymorphism. The same can be said regarding the next higher level, that of the genus. 